\title{
The Local Group dwarf irregular galaxy IC 1613
}

\section{The young stellar content}

\author{
L. Georgiev ${ }^{1}$, J. Borissova ${ }^{2}$, M. Rosado ${ }^{1}$, R. Kurtev ${ }^{3}$, G. Ivanov ${ }^{3}$, and G. Koenigsberger ${ }^{1}$ \\ 1 Instituto de Astronomía, Universidad Nacional Autónoma de México, México \\ e-mail: georgiev@astroscu.unam.mx,margarit@astroscu.unam.mx and gloria@astroscu.unam.mx \\ 2 Institute of Astronomy, Bulgarian Academy of Sciences, 72 Tsarigradsko chaussèe, BG-1784 Sofia, Bulgaria \\ e-mail: jura@haemimont.bg \\ 3 Department of Astronomy, Sofia University, BG-1164 Sofia, Bulgaria \\ e-mail: kurtev@phys.uni-sofia.bg and ivanov@phys.uni-sofia.bg
}

Received March 26; accepted July 1, 1998

\begin{abstract}
We obtained $U$-band photometry of 1500 stars and $B V$-band photometry of 3100 stars in a field centred on the HII regions of the northeast sector of the dwarf irregular galaxy IC 1613 - a member of the Local Group. The ages of the stellar populations were determined from the $\left((B-V)_{0}, M_{V}\right)$ color-magnitude using the isochrones from Padua's library. The stellar populations were split into three groups: the group of the youngest stars with ages of between 5 and $20 \mathrm{Myr}$, the group of intermediate stars with ages of $200-300 \mathrm{Myr}$ and the group of old stars with ages up to 10 Gyr. The slope of the Stellar Initial Mass Function $(I M F)$ for blue stars is $\Gamma=-2.0 \pm 0.1$ which is similar to that of other nearby galaxies. We investigated the stellar content of nine Hodge associations as well as that of seven new OB associations determined by cluster analysis. The total $U, B$ and $V$ magnitudes of eight Hodge open clusters and two new cluster candidates are given and there are six new possible nonstellar objects reported. The latter are most probably distant galaxies visible through the disk of IC 1613, which confirms the original suggestion of Baade that IC 1613 contains no bright globular clusters.
\end{abstract}

Key words: galaxies: individual: IC 1613 - galaxies: stellar content - galaxies: star clusters and associations

\section{Introduction}

The stellar content investigations of the Local Group of galaxies allow us to study in fine detail the star formation

Send offprint requests to: J. Borissova history of these systems. They also provide important verification information for the stellar evolution theory.

IC 1613 is a faint irregular member of the Local group. The galaxy was discovered by Wolf (1906) with the 16-inch Bruce refractor at Heidelberg. Baade (1928) using plates taken with the 16-inch Bergedorf reflector classified it as a Magellanic-Cloud-type galaxy. Baade (1963) and Sandage (1971) investigated variable stars, brightest stars and HII regions in the galaxy. Hodge (1978) published photoelectric and photographic surveys of its structure and stellar content. He identified twenty OB associations and pointed out the absence of bright globular clusters and the remarkably low number of stellar clusters. More recently, in 1988 Wendy Freedman presented $B, V, R$ and $I$ stellar photometry of over 2000 stars in two fields in IC 1613 from CCD frames obtained on the Canada-France-Hawaii Telescope. As pointed out by Freedman (1988a) "The disk of IC 1613 is seen to contain stars with a range of ages: 1 . young stars indicated by a distinctive plume of luminous blue stars, yellow supergiants, Cepheids, and a smaller number of red supergiant candidates at comparable luminosities, 2. intermediate-age stars, typified by very red extended asymptotic-giant-branch stars and carbon stars and finally, 3. a population consistent with that of an old, red giant population...". Saha et al. (1992) confirmed the presence of old type population by discovering the fifteen RR Lyrae stars in the field of IC 1613. There are several studies concerning HI (Lake \& Skillman 1989; Wenstpfahl et al. 1996). The rotation curve of IC 1613 shows evidence of dark matter in the outer parts. There is also evidence that the A10 Hodge association (Hodge et al. 1991) which is in the HII region has the youngest star ages among the rest. Eight W-R candidates are suspected (Armandroff \& Massey 1985) and one of them - WO3 - is surrounded by nebulae (Kingsburgh \& Barlow 1995). Finally IC 1613 is 
the only dwarf galaxy with a strong X-ray source detected (Helfand 1996) to be located close to the most luminous Oxygen Wolf-Rayet star.

This paper presents a deep $U B V$ photometry of the selected H-alpha emission area of IC 1613 as well as detailed investigations of its young stellar content.

\section{Observations and data reduction}

The observations of IC 1613 were carried out on the 2m Ritchey-Chretien telescope of the Bulgarian National Astronomical Observatory. A set of CCD frames in the $U B V$ system was taken on October 9, 1997 with a Photometrics $1024 \times 1024$ camera.

The seeing during the observations was about 1 arcsec with stable and very good photometric conditions. The scale at the cassegrain-focus CCD was 0.33 arcsec/pixel and the observing area was a $5.6 \times 5.6$ arcmin field centered on the HII regions in the northeast sector of the galaxy. The frames were obtained with total exposure times of $2700 \mathrm{~s}, 1800 \mathrm{~s}$ and $1800 \mathrm{~s}$ in the $U, B$ and $V$ filters, respectively. The mean airmass over the duration of each exposure was between 1.2 and 1.3. Two standard fields of NGC 1502 (Reimann \& Pfau 1987) were taken before and after the observations.

The standard IRAF data reduction package was used to carry out the basic CCD bias reduction and the flat field correction. The stellar photometry of the frames was done using DAOPHOT II (Stetson 1993). This package is widely used and besides Stetson's own description there are numerous references describing its application in detail (see for example Gallart et al. 1996a). The total number of measured stars is 1500 in $U, B$ and $V$ filters and 3100 in $B$ and $V$ respectively.

Fifteen to twenty relatively bright and isolated stars were chosen for each frame in order to obtain the aperture corrections and to transform the PSF magnitudes to the instrumental ones.

The atmospheric extinction for the observation night was calculated separately for each filter. The instrumental values were then corrected for extinction and transformed to the standard $U B V$ system using standard stars in the field of NGC 1502. The zero-point errors of the transformation were $0.01 \mathrm{mag}$ in $U, 0.02$ in $B$ and 0.04 in $V$. The magnitude residuals of the photometric transformation for the standard stars as a function of the $U, B$ and $V$ magnitudes are shown in Fig. 1.

The four photometric standard stars from the list of Sandage (1971) available in the field were transformed to the standard system so that we could check the zero point of our calibration on in-field data. One of them $-18 \mathrm{c}-$ displayed large differences of about $0.2 \mathrm{mag}$ in both $B$ and $V$ and taking into account its relative faintness (Sandage's magnitude in $B$ is 23.18) we removed it from the calculations. The average differences for the other three stars are 0.02 in $V,-0.02$ in $B$ and 0.05 in $U$. In Fig. 1 these stars are marked by crosses. This inaccuracy was however estimated to be within the standard photometry error and thus could not seriously affect the results of our study.

We have compared our data with the photometry of Freedman (1988a) in order to check for the presence of systematic effects. The residuals in the measurements of stars in common between the two studies are plotted in Fig. 2 as a function of the magnitudes and colors derived in the present investigation. As can be seen by inspection of these diagrams, no systematic trend is present in the $V$ magnitudes and $B-V$ colors. The mean differences are 0.03 in $B-V$ and 0.01 in $V$.

\section{Error analysis and completeness}

\subsection{Photometric errors}

In order to estimate the internal accuracy of our CCD measurements we used the formal errors from the DAOPHOT package and as additional error estimates we used the magnitude differences from experiments with artificial stars. Table ?? lists the derived average standard deviations for successive intervals of one magnitude in the $U, B$ and $V$ frames. As can be seen we have large errors $(>0.15)$ at the faint magnitudes: $U>22.5, B>23.5$ and $V>24.0$. The standard errors from all stars vs. magnitude are displayed in Fig. 3.

Table 1. The mean standard deviations

\begin{tabular}{cccc}
\hline Magnitude & $U$ & $B$ & $V$ \\
\hline $16.5-17.5$ & 0.005 & 0.003 & 0.004 \\
$17.5-18.5$ & 0.009 & 0.005 & 0.004 \\
$18.5-19.5$ & 0.026 & 0.011 & 0.011 \\
$19.5-20.5$ & 0.027 & 0.014 & 0.017 \\
$20.5-21.5$ & 0.048 & 0.022 & 0.025 \\
$21.5-22.5$ & 0.101 & 0.041 & 0.038 \\
$22.5-23.5$ & 0.260 & 0.091 & 0.072 \\
$23.5-24.5$ & - & 0.201 & 0.157 \\
$24.5-25.5$ & - & 0.380 & 0.356 \\
\hline
\end{tabular}

The artificial star simulations necessary for the completeness correction can also be used to determine the measurement errors. A set of artificial stars randomly placed on the original frames were re-determined in the same manner as the program stars. The "input-output" differences in $B$ and $V$ magnitudes between "added" and "recovered" artificial stars as a function of the measured output magnitude are shown in Fig. 4. The mean errors of the "input-output" differences in $B$ and $V$ magnitudes are 0.025 and 0.024 respectively. 

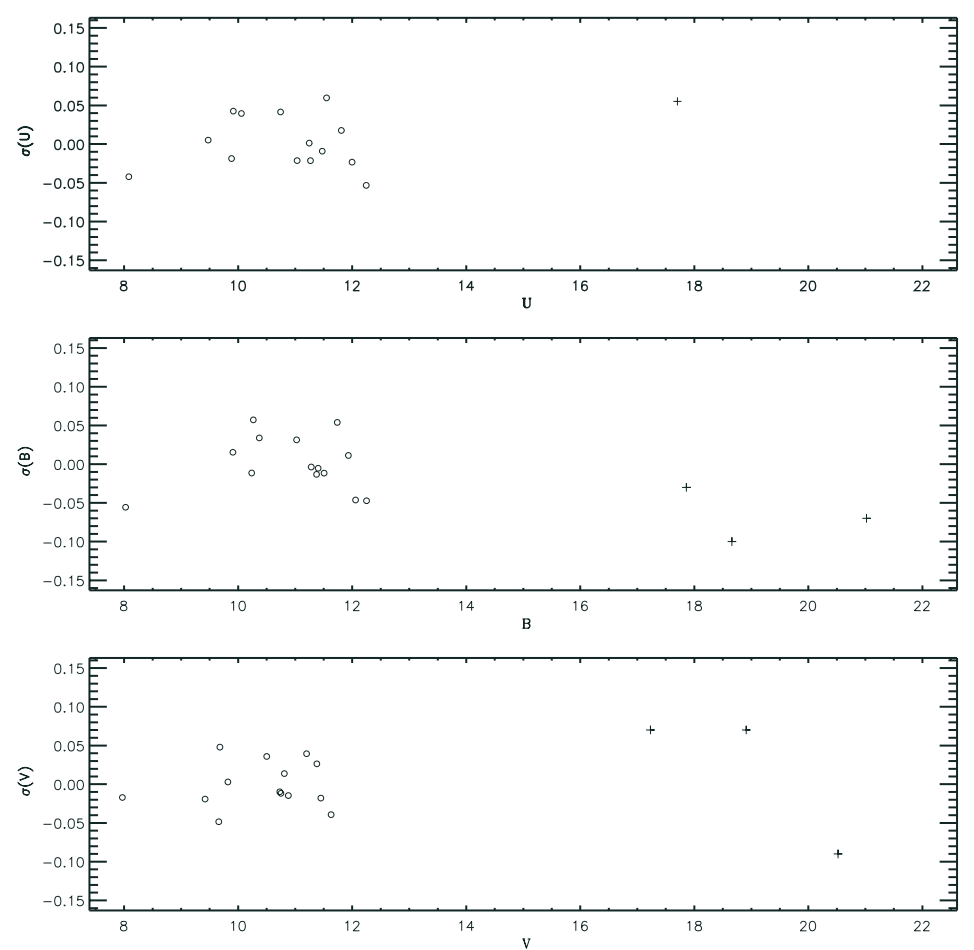

Fig. 1. The magnitude residuals of the photometric transformation at the standard stars as a function of the $U$ (upper panel), $B$ (medium panel) and $V$ (lower panel) standard magnitudes. The photoelectric photometric standards from Sandage (1971) are represented by crosses
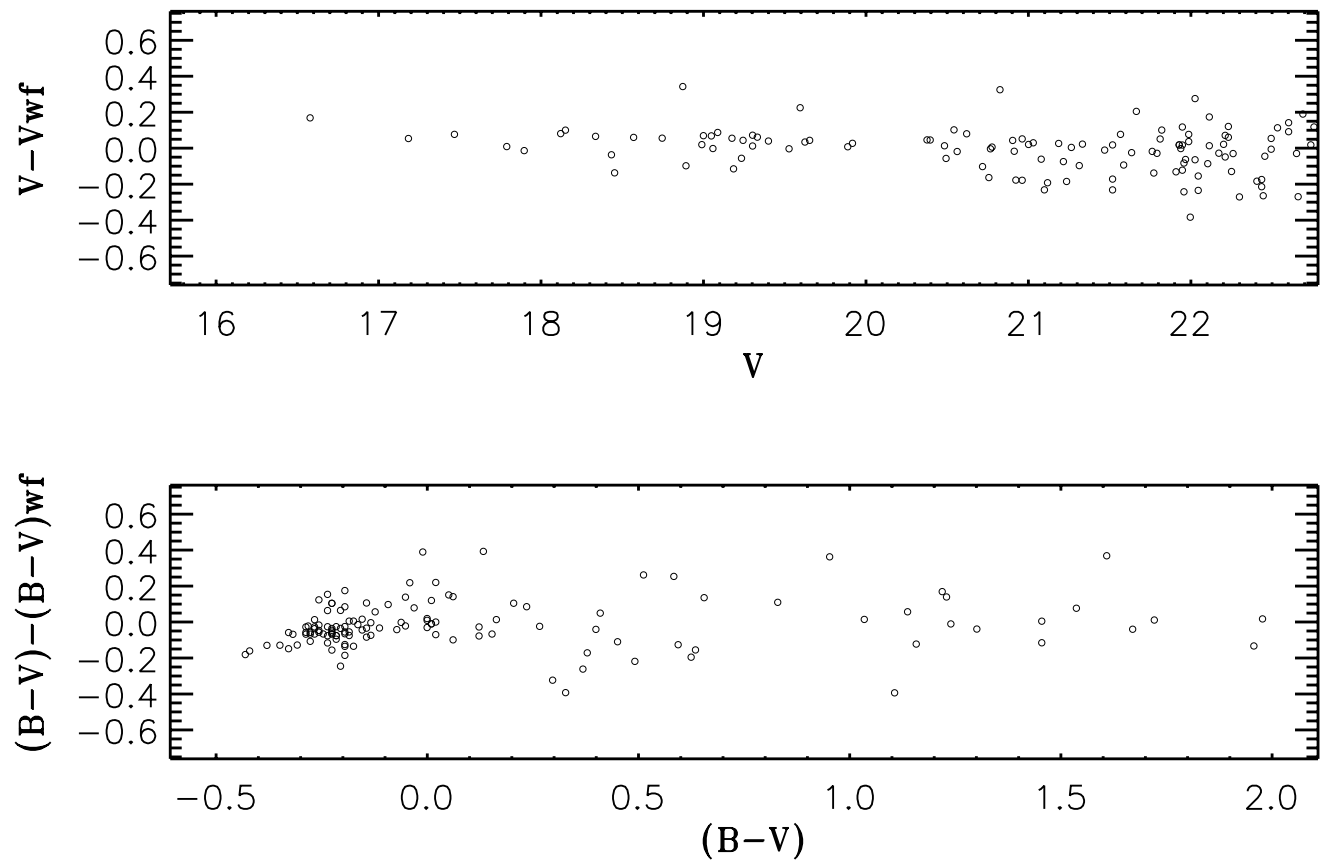

Fig. 2. Comparison of the $V$ magnitudes and $B-V$ colors derived here with the photometry of Freedman (1988a). The comparison is in the sense of this work minus Freedman (1988a) 

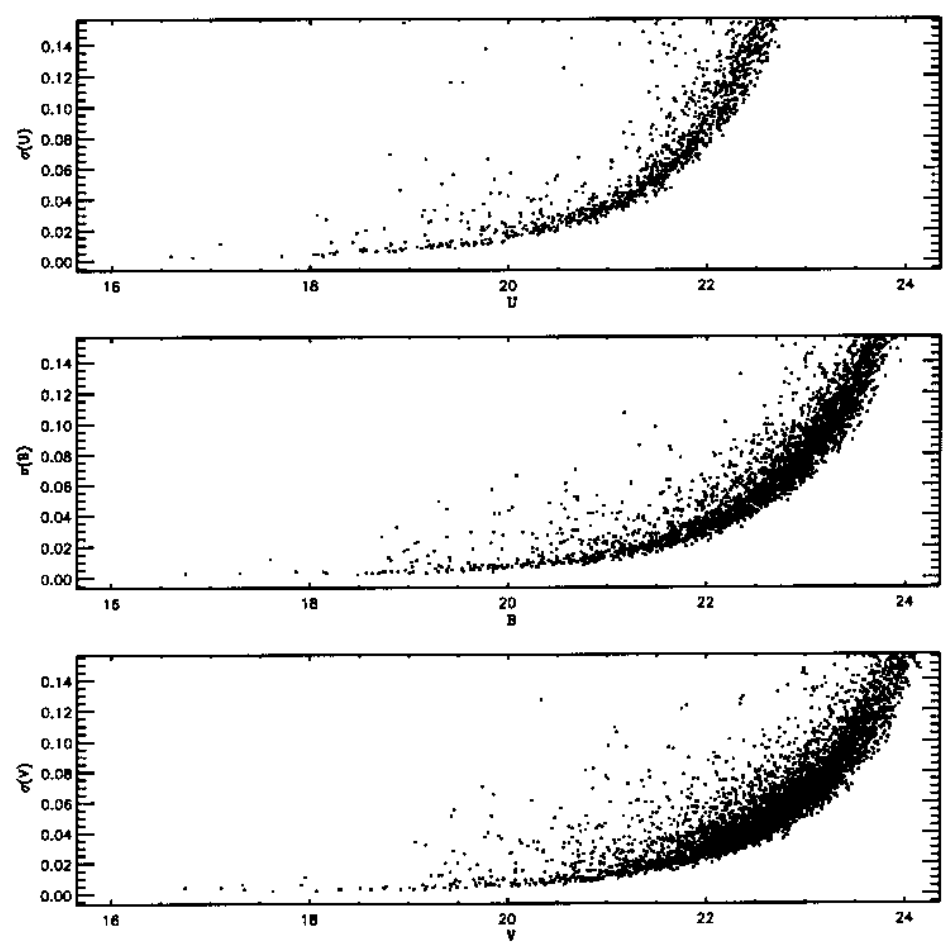

Fig. 3. Internal errors of the CCD photometry
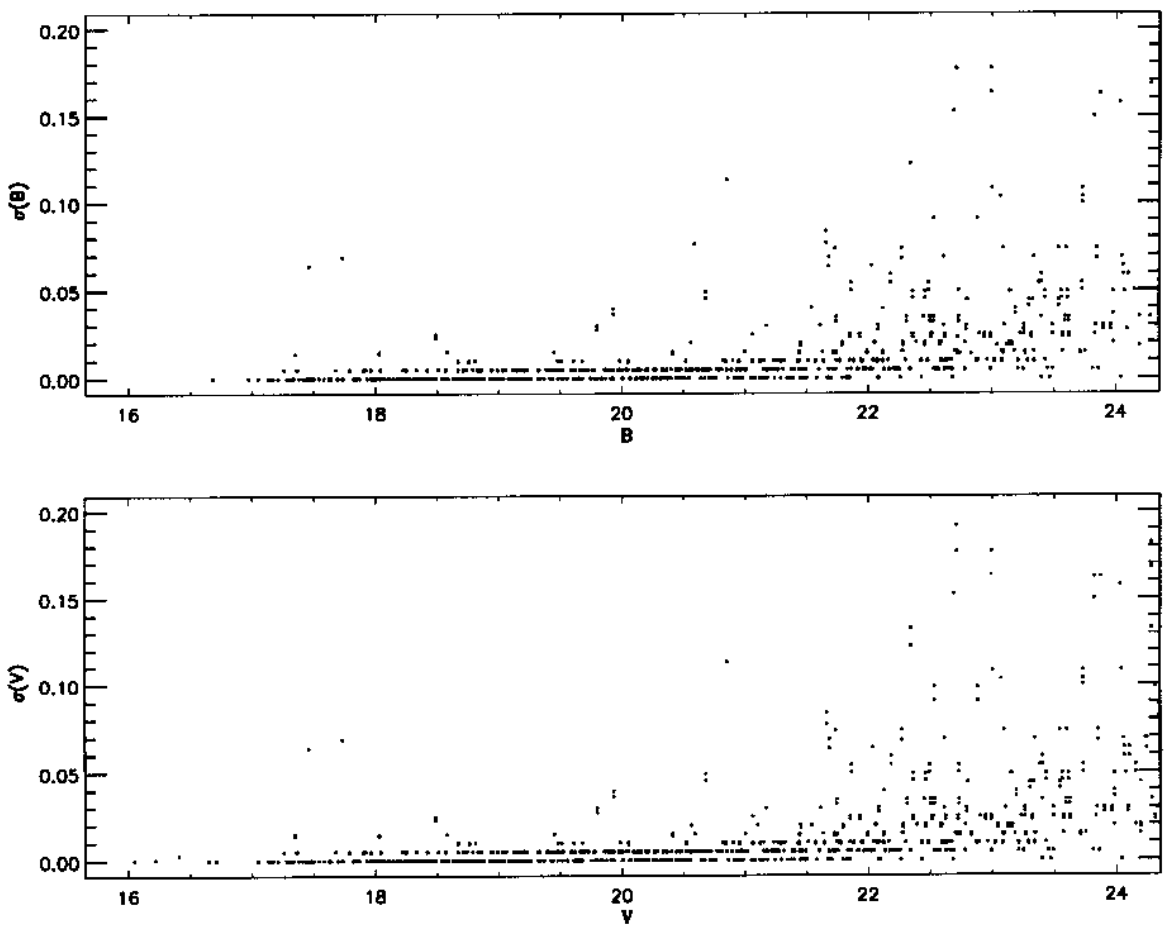

Fig. 4. The "input-output" differences in $B$ and $V$ magnitudes as a function of the measured output magnitude 


\subsection{Completeness correction}

The last step in our data reductions was to determine the completeness functions in the $B$ and $V$ filters. We used the artificial star technique (Stetson \& Harris 1988; Stetson 1991) creating series of artificial frames by means of the ADDSTAR routine in DAOPHOT II which were rereduced in the same manner as the original frames. The completeness correction derived as the ratio of added to recovered artificial stars was then fitted by a cubic spline. The completeness function is listed in Table 2. We consider our sample complete up to $22.5 \mathrm{mag}$ in both $B$ and $V$ filters. In Cols. 4 and 5 as independent completeness estimation the magnitude distributions for the stars in $U$ and $V$ filter binned by 0.5 are given. The turnover interval in $U$ filter is at $U=21.5 \mathrm{mag}$ which we will consider as a completeness limit in this filter. The completeness magnitudes in $B$ and $V$ agree with the values determined by artificial stars.

Table 2. Completeness function

\begin{tabular}{cccrr}
\hline Magnitude bin & $F(B)$ & $F(V)$ & $N_{U}$ & $N_{V}$ \\
\hline $16.5-17.0$ & 1.00 & 1.00 & 2 & 2 \\
$17.0-17.5$ & 1.00 & 1.00 & 1 & 3 \\
$17.5-18.0$ & 1.00 & 1.00 & 2 & 3 \\
$18.0-18.5$ & 1.00 & 1.00 & 16 & 5 \\
$18.5-19.0$ & 1.00 & 1.00 & 18 & 11 \\
$19.0-19.5$ & 1.00 & 1.00 & 34 & 27 \\
$19.5-20.0$ & 1.00 & 1.00 & 52 & 44 \\
$20.0-20.5$ & 0.98 & 0.99 & 63 & 74 \\
$20.5-21.0$ & 0.97 & 0.98 & 89 & 137 \\
$21.0-21.5$ & 0.96 & 0.97 & 165 & 231 \\
$21.5-22.0$ & 0.94 & 0.96 & 238 & 551 \\
$22.0-22.5$ & 0.90 & 0.94 & 204 & 725 \\
$22.5-23.0$ & 0.69 & 0.75 & 61 & 682 \\
$23.0-23.5$ & 0.32 & 0.44 & 26 & 398 \\
$23.5-24.0$ & 0.12 & 0.38 & - & 157 \\
$24.0-24.5$ & 0.07 & 0.18 & - & 45 \\
\hline
\end{tabular}

\subsection{Field star contamination}

It is obviously necessary to check the level of field star contamination in the color magnitude diagram before discussing its detailed structure. Based on the Bahcall \& Soneira (1980) model of the Galaxy, Ratnatunga \& Bahcall (1985) have predicted the field star densities in the direction of IC 1613. Table ?? gives the number of objects expected per bin of magnitude and color in the area covered by our observations. In summary the total number of expected field stars in our field is 47 , while we can expect approximately two stars brighter than 19 mag in $V$ and $(B-V)<0.8$. Field stars therefore should not seriously affect the structure of the CMD and no corrections for field contamination have been applied to the IC 1613 data.

\section{General morphology of the IC 1613}

\subsection{Color-magnitude diagrams}

Figures 5 and 6 show the $(U-B, V)$ and $(B-V, V) \mathrm{CM}$ diagrams obtained for IC 1613 . To construct the $(U-B, V)$ color-magnitude diagram we selected only the stars with photometric errors not larger than 0.15 in all filters while in the second $(B-V, V)$ diagram are plotted all stars measured in our field.

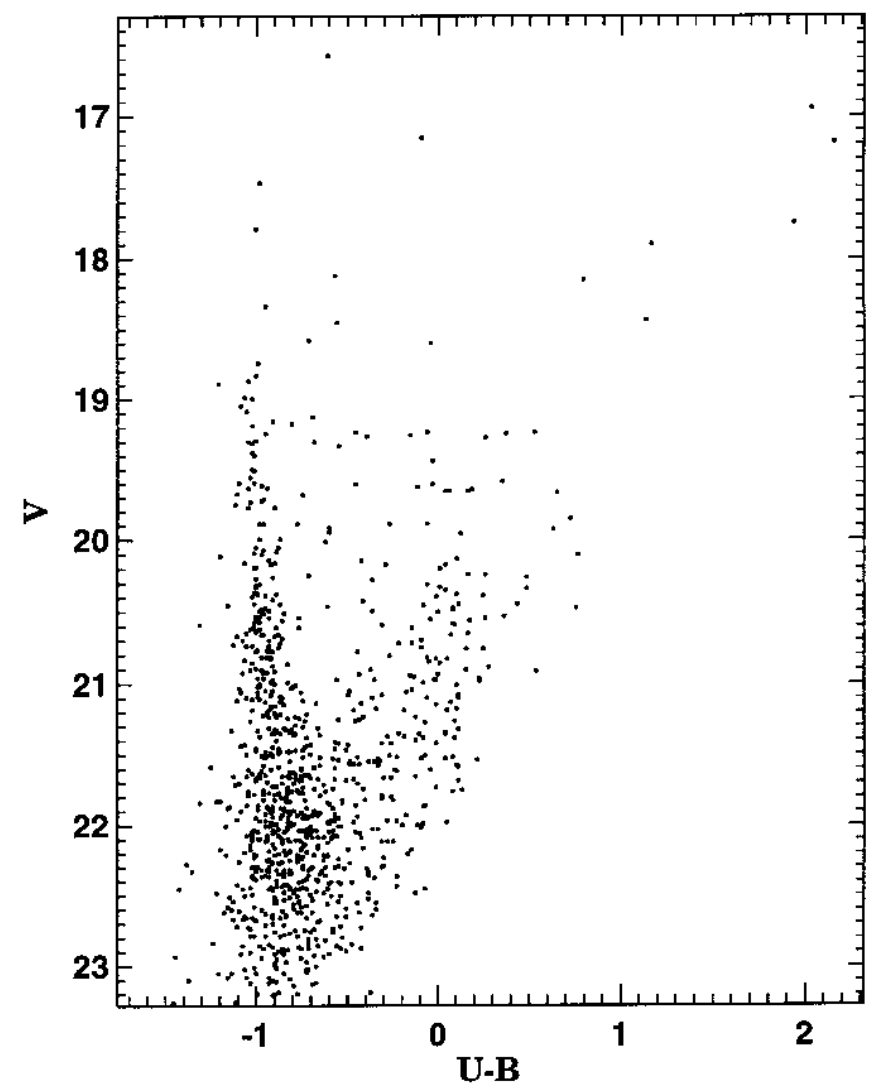

Fig. 5. $(U-B, V)$ color - magnitude diagram obtained for IC 1613. Only stars with photometric errors larger than 0.15 in all filters are plotted

As pointed out by Freedman (1988a), "A first glance at both color - magnitude representations reveals immediately the presence of a bright, young, blue population, an intermediate-color population, and a red population composed of both bright, young supergiants and a fainter red giant population".

All previous photometric works on IC 1613 - Baade (1963), Sandage (1971) and Freedman (1988a) pointed out the presence of young, intermediate and old age population using only the general morphology of the colormagnitude diagrams in different filters. In this work we 
Table 3. Expected numbers of field stars towards IC 1613

\begin{tabular}{lccccc}
\hline \multicolumn{1}{c}{ Color range } & $15<V<17$ & $17<V<19$ & $19<V<21$ & $21<V<23$ & $23<V<25$ \\
\hline$B-V<0.8$ & 0.78 & 1.31 & 2.94 & 2.69 & 0.94 \\
$0.8<B-V<1.3$ & 0.90 & 0.97 & 1.06 & 2.88 & 3.44 \\
$B-V>1.3$ & 0.34 & 1.63 & 4.39 & 8.15 & 14.4 \\
Total & 2.00 & 3.76 & 8.46 & 13.7 & 18.8 \\
\hline
\end{tabular}

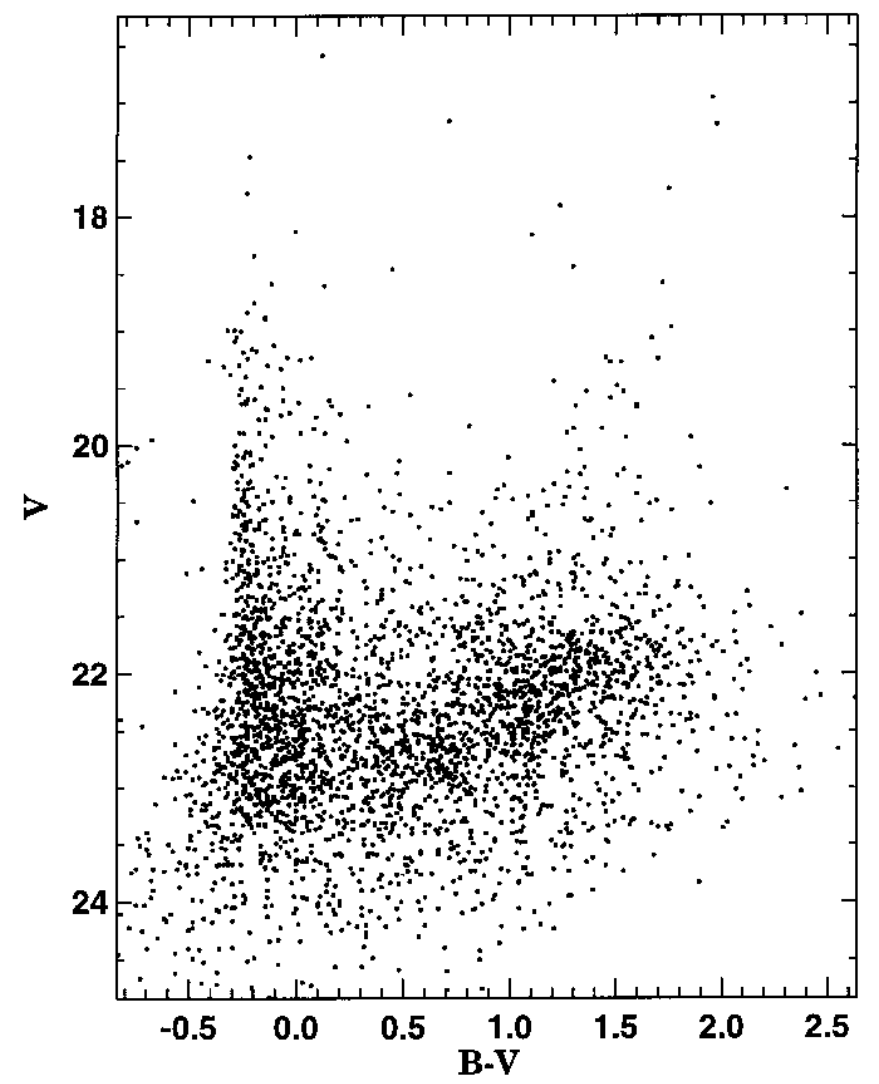

Fig. 6. $(B-V, V)$ color - magnitude diagram for IC 1613

present a detailed investigation of the young stellar content based on the comparison with theoretical isochrones and $\mathrm{H}-\mathrm{R}$ diagrams.

\subsection{Differential reddening}

Our first step in the morphology analysis of IC 1613 was to determine the differential (internal and foreground) reddening across the galaxy. Sandage (1971) obtained $E(B-V)<0.03$ from 7 photoelectrically measured stars. Freedman (1988a) determined $E(B-V)=0.04$. The latest $E(B-V)$ value for IC 1613 derived from spectral measurements is 0.07 (Kingsburgh \& Barlow 1995). Our field of investigation is centred on the HII regions of the galaxy and we do not exclude the presence of a higher internal reddening in this area. We used Johnson's $Q$-parameter technique to measure the reddening $(Q=$ $(U-B)-0.72(B-V)$ is a reddening-free quantity), cal- culating the $Q$ values for all stars belonging to the main sequence (luminosity class $V$ ). These stars stand at $V$ magnitudes between 20.0 and 23 and at $U-B$ colors between -1.2 and -0.5 (see Fig. 5 ). The $E(B-V)$ for each star was calculated by means of the equations given in Massey et al. (1995a). We will assume that the "mean" value for $E(B-V)$ in this area of IC 1613 is the average of the above determined individual $E(B-V)$ values. Our final value for $E(B-V)$ is 0.06 with $\sigma=0.02$. We thus confirm the very low reddening of IC 1613 estimated by Sandage (1971) and Freedman (1988a).

Figure 7 shows the $(B-V, U-B)$ "unshifted" colorcolor diagram of IC 1613. This diagram contains only stars with photometric error less than 0.15 . The fiducial locus of the stars of luminosity class $V$ and that of luminosity class $I a b$ (dashed line) were superimposed (Schmidt-Kaler 1982). The O-B9 stars closely follow the sequences of luminosity class $V$ and $I a b$ at $U-B<0.2$ and $B-V<0.2$. There are some stars around $(U-B)=0$ which follow the fiducial line of the $V$ luminosity class and are probably foreground Galactic stars. The two stars located close to $(B-V)=2,(U-B)=2$ are the variable bright red supergiants V32 and V38 (Sandage 1971).

\subsection{Comparisons with theoretical isochrones}

Figure 8 shows the $\left((B-V)_{0}, M_{V}\right)$ color magnitude diagram with superimposed isochrones from Padua's library (see Bertelli et al. 1994). We chose for our comparison metallicity $z=0.004$ which corresponds to the metallicity value measured for IC 1613 by Kingsburgh \& Barlow (1995). The adopted distance modulus is 24.20 (Freedman 1988a; Saha et al. 1992), while for the extinction we used $E(B-V)=0.06$ as has been determined by our analysis. The isochrones fit clearly shows the presence of stars in a wide variety of ages. The age of the blue stars is between 5 and 20 Myrs. Most of the yellow to red stars fainter than $M_{V}=-4.0$ are roundly matched by the post main-sequence part of the 60 Myrs to 250 Myrs isochrones. The last isochrone in Fig. 8 represents the Vanden Berg (1997) isochrones data set for Population II stars with $z=0.004$ and an age of 10 Gyr. The presence of such old stars is not unusual and has been found in other Local dwarf galaxies. Gallart et al. (1996a-c) found in NGC 6822 strong evidence for the presence of a considerable amount of old stars. The "most striking" features presented in their CMD are the so called "red-tangle" and "red-tail" - 


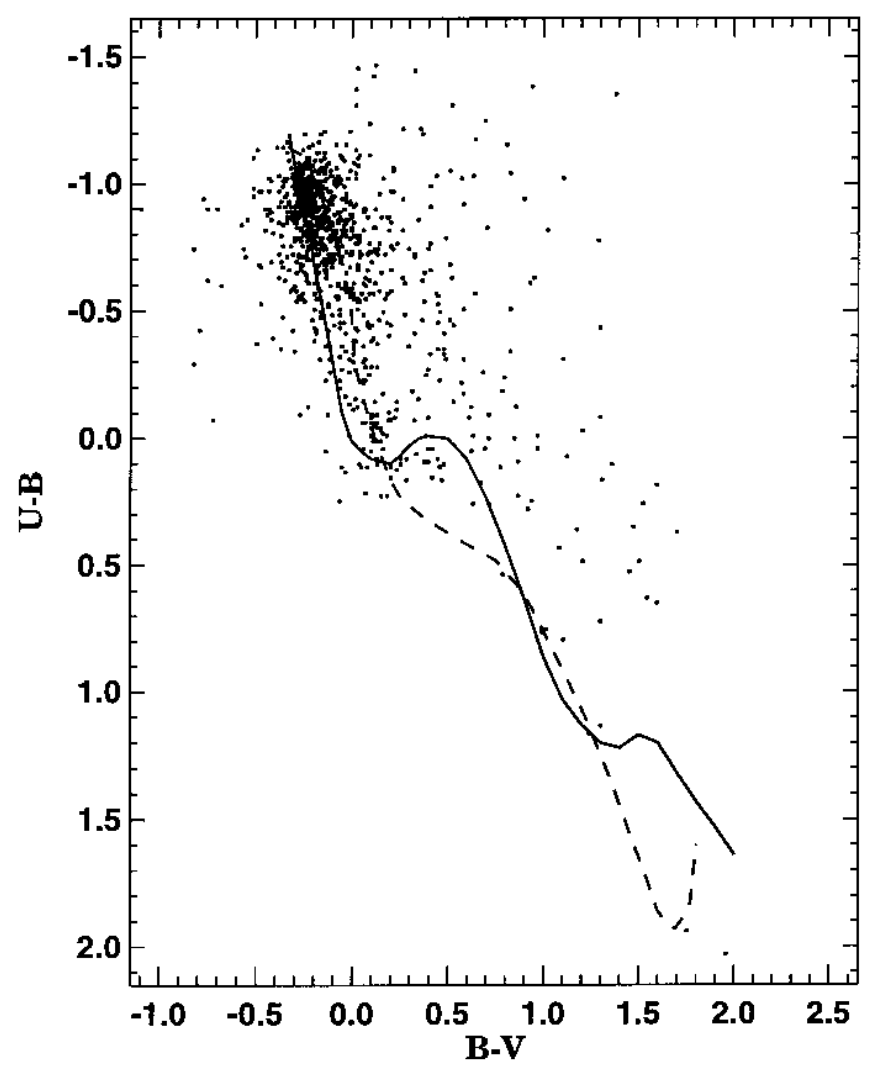

Fig. 7. $(U-B, B-V)$ color-color diagram obtained for IC 1613 . Only stars with photometric errors less than 0.15 in all filters are plotted. The fiducial locus of stars of luminosity class $V$ and of luminosity class $I a b$ (dashed line) are superimposed

the crowded clumps in the lower red part of their $(V-I, V)$ diagram. The same features are less visible in our CMD for two reasons: such red stars are more evident in red bands $(I$ and $R$ ) and in this part of the CMD the completeness of our sample is relatively low (see Table 2 ).

\section{4. $H-R$ diagram}

To plot the stars on the theoretical $\mathrm{H}-\mathrm{R}$ diagram we need to determine their effective temperatures $\left(T_{\text {eff }}\right)$ and bolometric corrections (BC). We followed closely the procedure of Massey et al. (1989, 1995a). We have no spectra for stars in this area of IC 1613 and we used the transformation equations given in Table 7 of Massey et al. (1995a) to derive $\log \left(T_{\text {eff }}\right)$ and $\mathrm{BC}$. We have assumed the slope factor of the reddening law to be $E(U-B) / E(B-V)=0.72$ as it is for our Galaxy, NGC 6822 and M33. Our attempt to determine the effective temperatures of stars, $(B-V)_{0}$ colors and bolometric correction with the improved numerical relation given in Flower (1996) yielded unrealistic $\log \left(T_{\text {eff }}\right)$ and $M_{\text {bol }}$ for very blue stars (with $\left.(B-V)_{0}<-0.2\right)$, so we preferred to use the calibration equations of Massey et al. (1995a) in the whole $B-V$ interval. Figure 9 shows $\left(\log \left(T_{\text {eff }}\right), M_{\text {bol }}\right)$ for stars with

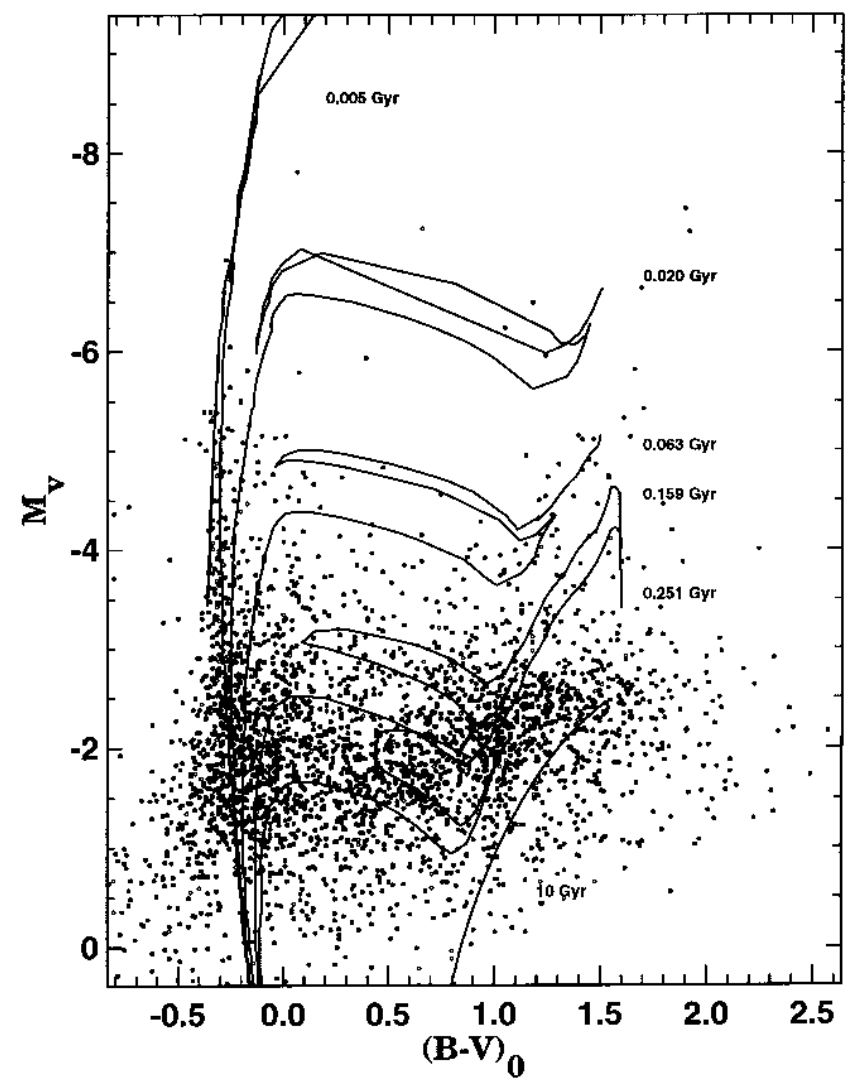

Fig. 8. $\left((V-B)_{0}, M_{V}\right)$ color-magnitude diagram for IC 1613. The adopted distance modulus and extinction are $(M-m)=$ 24.20 and $E(B-V)=0.06$. The isochrones from Padua's library with $z=0.004$ are superimposed. Labels stand for the corresponding ages in Gyr. The last isochrone represents the 10 Gyr Population II stars (Vanden Berg 1997)

photometric errors less than 0.15 mag. The evolutionary mass tracks from Charbonnel et al. (1993) for $z=0.004$ were superimposed on the same plot.

We have used the distance modulus as in the isochrone comparison. The individual stellar reddening was calculated using the $Q$-method for blue main sequence stars and blue supergaints. The mean $E(B-V)=0.06$ was adopted for all other stars. Equations of Massey et al. (1995a) require knowledge of star's luminosity class. While this is an unknown without spectroscopic classifications we used the calibration of the minimum $M_{V}$ as a function of $Q$ given in Parker \& Garmany (1993) (see their Eq. (3)) to separate supergaints from dwarfs.

As can be seen in Fig. 9 most of the blue stars are located quite close to the ZAMS, including the stars of $60-85 M_{\odot}$. The stars with masses lower than $\left(5 M_{\odot}\right)$ systematically deviate to lower effective temperatures than those on the ZAMS. We have a large incompleteness in the $U$ filter for stars with $M_{\text {bol }}<-3.0$, so we consider that this is a systematic deviation resulting from a combination of incompleteness and uncertain $Q$-parameter. Some stars in the $\mathrm{H}-\mathrm{R}$ diagram fall far to the left of the ZAMS. 
A careful check of these stars shows that they have relatively large photometric errors and/or very low $Q$ values. Generally specking the errors in theoretical $\mathrm{H}-\mathrm{R}$ diagrams are combination of photometric errors (especially in the $U$ magnitudes, which can lead to incorrectly high temperatures and bolometric corrections), transformation equations, reddening determination and adopted distance modulus.

The red part of the $\mathrm{H}-\mathrm{R}$ diagram contains five very red and luminous supergiants with masses between 12 and $40 M_{\odot}$, a large amount of low-mass yellow stars and red giants from the tip of the RGB.

\subsection{Variable stars}

With no spectra available we calculated the effective temperatures and $M_{\text {bol }}$ for all available variable stars in our field in order to check if the red supergiants were correctly located on the $\mathrm{H}-\mathrm{R}$ diagram. They are marked in Fig. 9 with open squares and listed in Table 4. Freedman (1988b) determines the light curves and periods for most of the Cepheids suspected by Sandage (1971). The Cepheids V10, V18 and V39 have periods of 4.07, 16.44 and 28.72 days respectively and as can be seen in our $\mathrm{H}-\mathrm{R}$ diagram they lie within the instability strip of the Cepheids. V22 is among the Cepheids with the longest periods having a period of 146 days and therefore the relatively high $M_{\text {bol }}$ for this star is not unusual. V32, V38, V40, V43 and V56 are red irregular variables suspected by Sandage (1971) and confirmed by Freedman (1988a). We can see that these stars are located correctly on our $\mathrm{H}-\mathrm{R}$ diagram. V8 and V21 are especially interesting from our point of view. V8 is an irregular variable (Carlson \& Sandage 1990) which varied from $B=21.3$ to $B=21.9$ over a 33 year interval. In our diagram this star is a blue one and has relatively high $M_{\text {bol }}$ and $T_{\text {eff }}$ (See Table 4). Sandage (1971) notes that V21 is an intermediate color irregular variable with a small amplitude. From 1929 to 1937 the star varied from $B=19.60$ to $B=20.34$ mag. The largest variations occurred between 1929 and 1932. The star remained relatively constant after that to within $0.2 \mathrm{mag}$ at $B=20$ mag. In 1984 (photometry of Freedman 1988a) the star was very blue with $B-V=-0.24$ and $V=20.44$. In our photometry based on 9.10.1997 observations the situation is the same - the star is very blue. The star is hot with $T_{\text {eff }}$ approx. $30000 \mathrm{~K}$, it is one of the most luminous stars with $M_{\mathrm{bol}}=-7.67$ and its mass is approximately $20 M_{\odot}$.

\subsection{The $I M F$}

From the isochrone fit shown in Fig. 8 we have selected the blue stars in the main sequence with equal age $(5-10 \mathrm{Myr})$ in order to construct the $I M F$. Following
Table 4. Variable stars

\begin{tabular}{lccccccr}
\hline Name & $X$ & $Y$ & $V$ & $B-V$ & $\log \left(T_{\text {eff }}\right)$ & $M_{\text {bol }}$ & $M_{V}$ \\
\hline V8 & 950.3 & 284.8 & 21.37 & -0.15 & 4.50 & -6.47 & -3.25 \\
V21 & 452.1 & 797.6 & 19.89 & -0.17 & 4.47 & -7.67 & -4.63 \\
V32 & 760.7 & 456.2 & 16.95 & 1.96 & 3.48 & -9.84 & -7.44 \\
V38 & 599.7 & 366.4 & 17.18 & 1.98 & 3.48 & -9.70 & -7.20 \\
V40 & 515.2 & 1004.0 & 18.96 & 1.76 & 3.53 & -7.07 & -5.43 \\
V43 & 449.2 & 999.7 & 17.75 & 1.75 & 3.53 & -8.22 & -6.64 \\
V56 & 567.4 & 807.2 & 17.90 & 1.24 & 3.64 & -6.84 & -6.49 \\
\hline
\end{tabular}

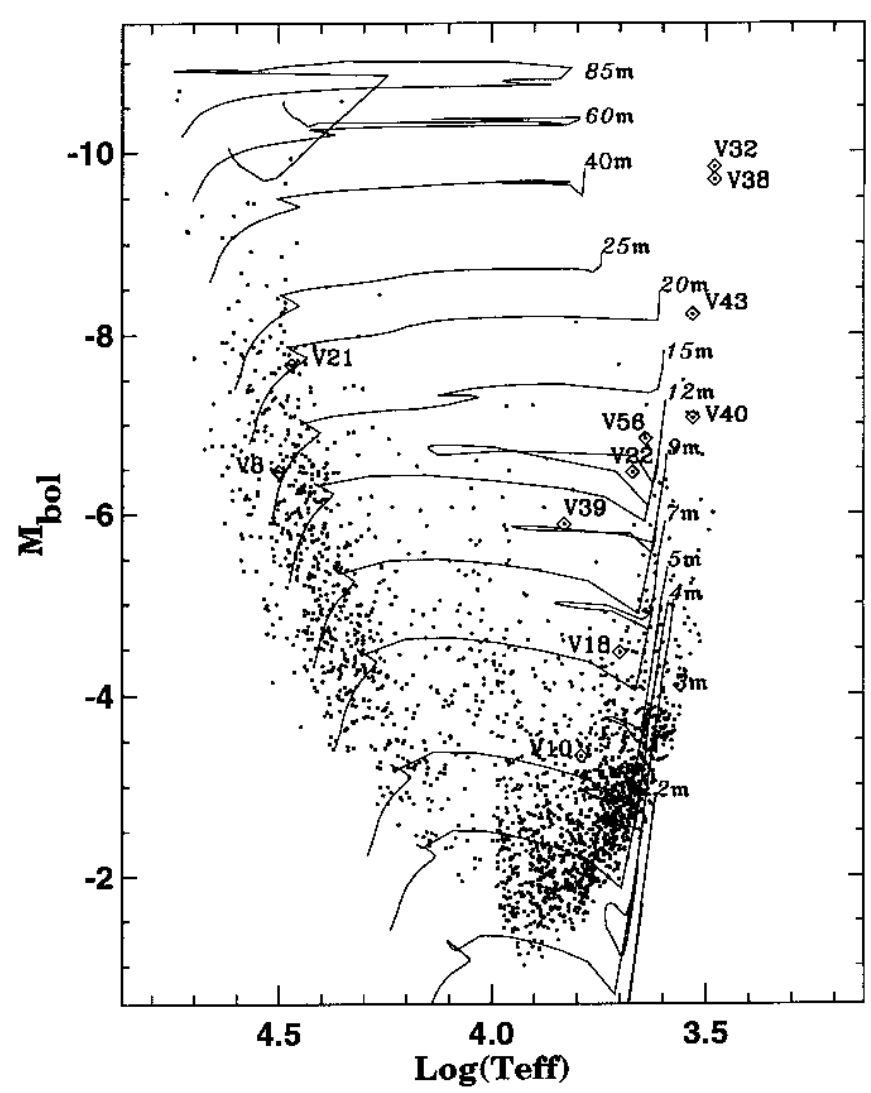

Fig. 9. H-R diagram for all stars in IC 1613. The evolutionary mass tracks from Charbonnel et al. (1993) for $z=0.004$ are superimposed on the same plot. Eleven variable stars are marked by open squares

again Massey et al. (1989, 1995a,b) we determine the slope $\Gamma$ of $I M F$ as:

$\Gamma=d \log \xi(\log m) / d \log (m)$

where $\xi(\log m)$ is the number of stars formed per logarithmic mass interval per unit area. The Salpeter (1955) slope is $\Gamma=-1.35$. To determine our $I M F$ we simply counted the stars that fall between each pair of mass tracks in Fig. 9 and normalized the count to the width of the bin and the area of the observed field. We left out the stars with masses less than $7 M_{\odot}$ when the incompleteness in our sample became significant. Our final slope is $\Gamma=-2.0$ with $\sigma=0.1$ (the uncertainty in the slope is the uncertainty in the least-squares fit to the $I M F)$. The $I M F$ 


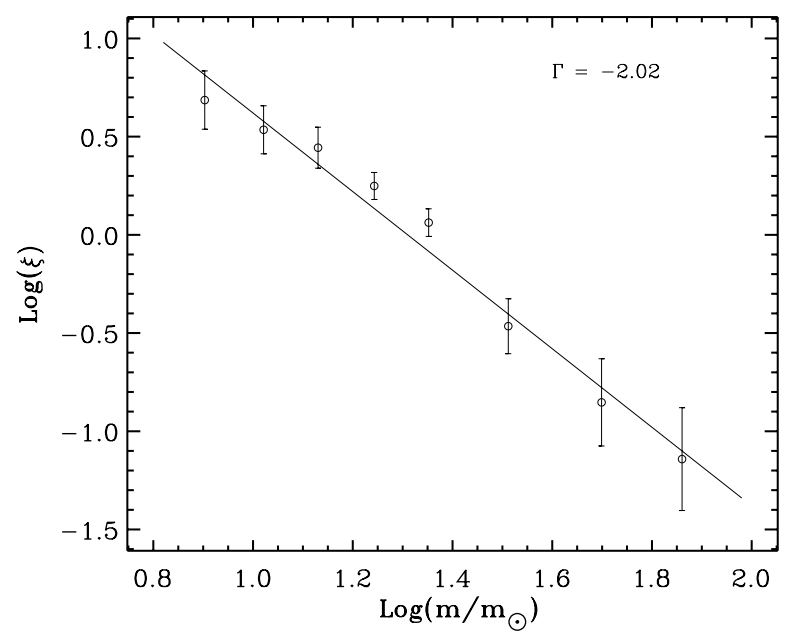

Fig. 10. The initial mass function for IC 1613. The error bars indicated statistical fluctuations

is shown in Fig. 10. If we exclude the highest mass bin $\left(M_{\odot}>60\right)$ where the statistical errors are largest and to avoid possible age differences the slope of $I M F$ became $\Gamma=-1.98$ with $\sigma=0.1$. No correction for unresolved multiple stars has been made.

The comparison with the $I M F s$ of other galaxies given in Haiman et al. (1994) (see their Table 2) shows that our result is consistent within the error range with the results for LMC, SMC and some of the M 31 associations. Salpeter $I M F s$ have been measured for intermediate mass stars in the Milky Way open clusters and associations (Phelps et al. 1993; Massey et al. 1995a,b). The IC 1613 formation therefore appears to be typical of star formation processes in other nearby galaxies.

\section{Associations}

OB associations are collections of recently formed stars which can be distinguished from the background of field stars by the clustering of bright, young $\mathrm{O}$ and $\mathrm{B}$ stars. In general they are not gravitationally bound systems and are short-lived features. For these reasons investigations of the OB associations can provide information about the formation and evolution of massive stars.

\subsection{Hodge associations}

Hodge (1978) identified twenty OB associations concentrated primarily in the core of the galaxy with a mean diameter of 164 pc. Freedman (1988a) presented colormagnitude diagrams for 11 of them and gave some age estimates using period-age relations for Cepheids. In our field of observations we have 9 of the Hodge associations - Nos. 10, 11, 12, 13, 14, 15, 17, 18 and No. 19. Figure 11 shows the $(V-B, V)$ color-magnitude diagrams of the
Hodge associations. The new plot of color-magnitudes diagrams shows well-defined main sequences with widths of typically 0.2, blue and red supergiants in A10, A11 and A14 and some intermediate age stars and red giant stars.

Figure 12 and Fig. 13 show the comparison with the isochrones from Padua's library (see Bertelli et al. 1994) and a $\left(\log \left(T_{\text {eff }}\right), M_{\text {bol }}\right)$ diagram. They were built in the same manner as in the previous section.

On the assumption that all the stars in each association have the same age we can determine the age of the associations following the method of Flannery \& Johnson (1982). The distance of the star $j$ of the association to a given isochrone was computed as:

$d_{j}^{2}=M I N_{i=1, \ldots, n}\left[\left(\frac{V_{j}-V_{i}}{\sigma_{V_{j}}}\right)^{2}+\left(\frac{(B-V)_{j}-(B-V)_{i}}{\sigma_{(B-V)_{j}}}\right)^{2}\right]$

where $V_{i}$ and $(B-V)_{i}$ are the different points of the isochrone given by different stellar masses. A mean distance of stars to the isochrone can be obtained by:

$\psi=\left(\sum_{j, \ldots, N} d_{j}\right) / N$

where $N$ is the number of stars. The minimum value of $\psi$ gives the most probable age of the association. Due to observational errors as well as a certain amount of inaccuracy in the estimation of $E(B-V)$ and $[\mathrm{Fe} / \mathrm{H}]$ the total external uncertainty of this method is about 2.5 Myr. The "most probable" age of the associations is given in Table 5, Col. 2. We would like to point out however that in most cases the $\psi$ value is rather high and it could indicate the presence of a spread of ages throughout the association. Nevertheless, as can be seen in Table 5 there are significant age differences among the associations which are generally larger than the uncertainty of the method. The youngest associations are A10 and A14 with ages of approx. 5 Myr while the oldest ones are A12, A18 and A19 with ages of approx. $20 \mathrm{Myr}$.

As can be seen in Fig. 12 there are some yellow and red stars in all associations, which have ages between 100 and 300 Myr. In the theoretical $\mathrm{H}-\mathrm{R}$ diagram (Fig. 13) they lie between 4 and $7 M_{\odot}$. We consider one small part of them to be field stars from our Galaxy (according to Table ?? in $V<21 \mathrm{mag}$ the expected number of field stars is approximately 30) and the rest to be field stars from IC 1613. To investigate the behavior of foreground stars we selected the "comparison field area" with the same mean diameter as the Hodge association - 164 pc and far from any known association. The color-magnitude diagram, the isochrone comparison and the $\mathrm{H}-\mathrm{R}$ diagram were built in the same way as in the associations and are shown in Fig. 14. As can be easily seen except for a few faint blue stars that lie on the main sequence most stars are faint, low-mass and 100 - 300 Myrs old.

In the upper part of the theoretical $\mathrm{H}-\mathrm{R}$ diagram (masses $>25 M_{\odot}$, Fig. 13) most of the associations except A12, A18 and A19 contain young massive stars which have 

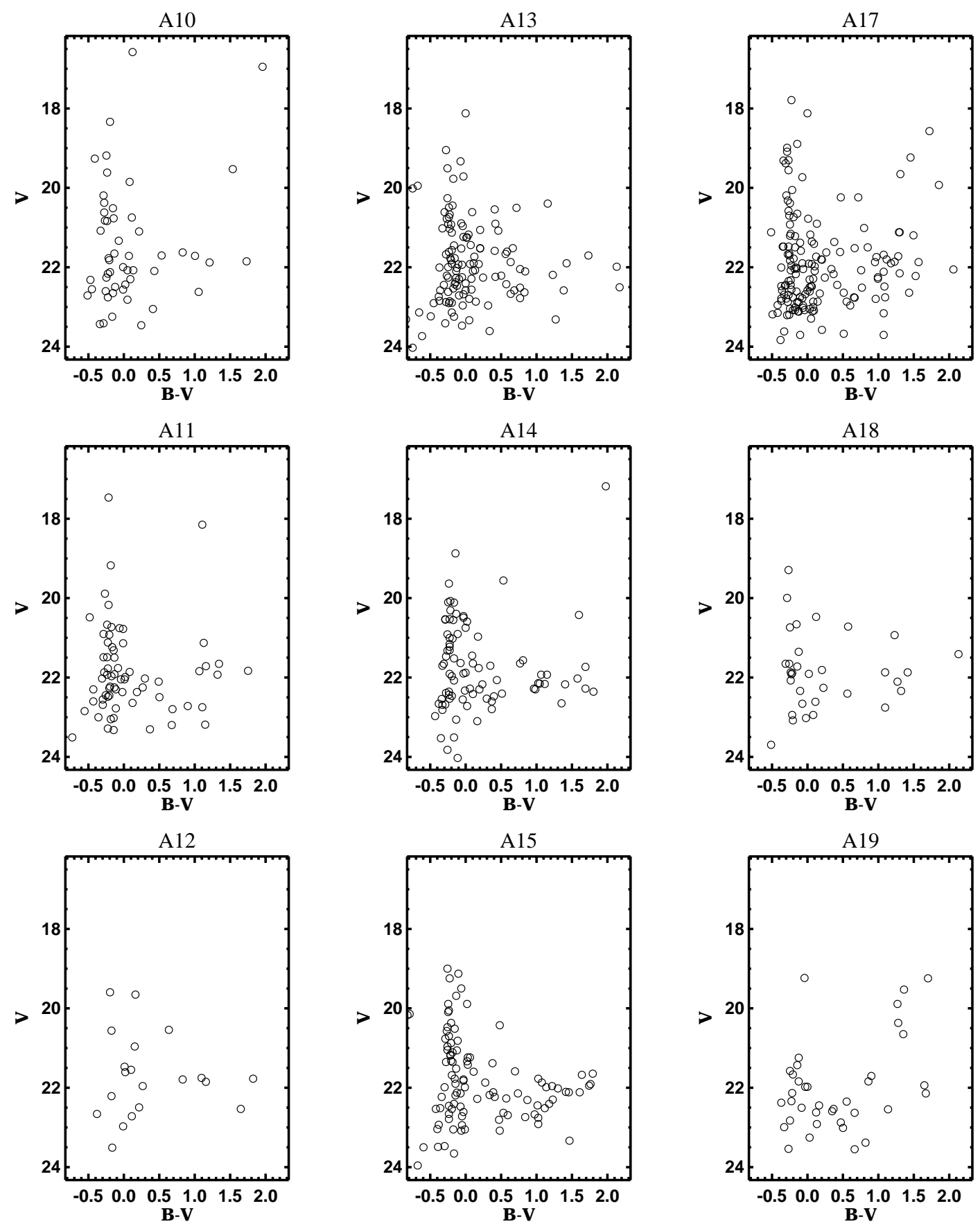

Fig. 11. $(B-V, V)$ color-magnitude diagrams for Hodge associations Nos. 10, 11, 12, 13, 14, 15, 17, 18 and No. 19

been born almost coeval. Following Massey et al. (1995) we tabulated the most massive stars still present in the associations in Table 5. The masses of the most massive stars are listed in Col. 3 and Col. 4 contains a number of stars with masses higher than $12 M_{\odot}$. The analysis of Table 5 shows that the associations containing the most massive stars are the youngest ones while the associations containing only low-mass stars are indeed the oldest.

Hodge (1978) included the brightest red supergiants V32 and V38 in the boundaries of A10 and A14. According to the paper of Massey (1998) they could be real members of those associations. V32 and V38 stand around $40 M_{\odot}$ (Fig. 13) and have very high $M_{\mathrm{bol}}-9.84$ and -9.70 respectively. On the other hand the age of both A10 and A14 is $5 \pm 2$ Myr. As Massey (1998) mentioned. "At 5 Myr we would find only WN stars and few if any RSGs, and at 7 Myr we would find only RSGs". The comparison with the masses and $M_{\mathrm{bol}}$ of the red supergaints in M 31, M 33 and NGC 6822 given by Massey (1998) shows 

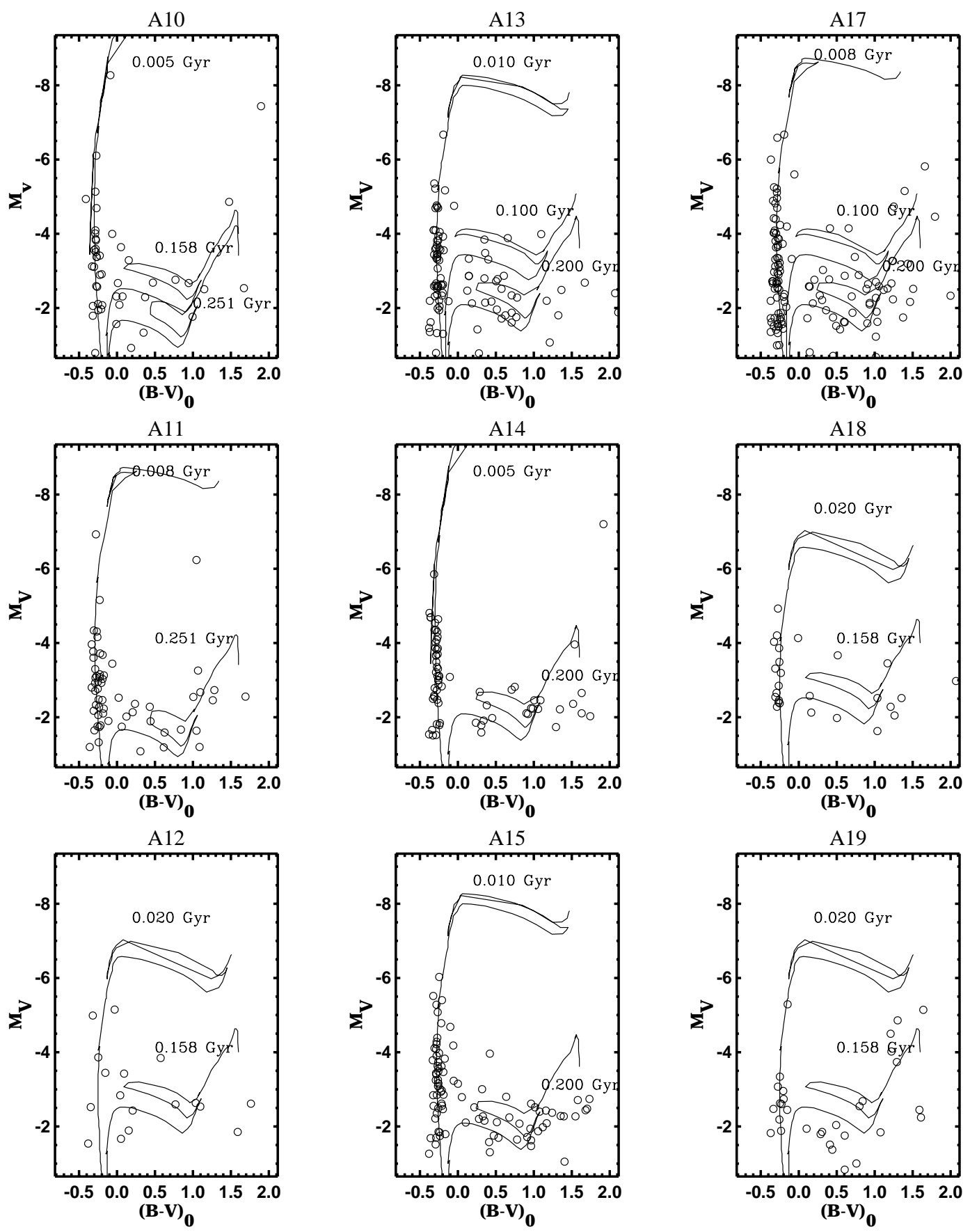

Fig. 12. $\left((V-B)_{0}, M_{V}\right)$ color-magnitude diagrams for Hodge associations. Isochrones from Padua's library with $z=0.004$ are superimposed

that the two supergaints in IC 1613 are similar to the red supergaints in NGC 6822.

Three Wolf-Rayet (WR) stars reported by Armandroff \& Massey (1985) - WR4, WR5 and WR8 are in our field of observations. These stars belong to the associations A10, A13 and A14 respectively and have following magnitudes and colors: WR $4-V=19.16, U-B=-0.39$, $B-V=-0.41 ;$ WR5 $-V=20.78, U-B=-1.00$, $B-V=-0.27 ; \mathrm{WR} 8-V=19.56, U-B=-1.80$,
$B-V=0.53$. The WR 8 is embedded in the supernova remnant S8 (Peimbert et al. 1988), which lead to the very unrealistic magnitude and colors. The presents of the WR stars in A10, A13 and A14 is in good agreement with youngest age of these associations.

It is not possible to calculate the $I M F$ for each association because of small association members and therefore large statistical errors. In order to improve the statistics we summarized Hodge associations in three groups. 

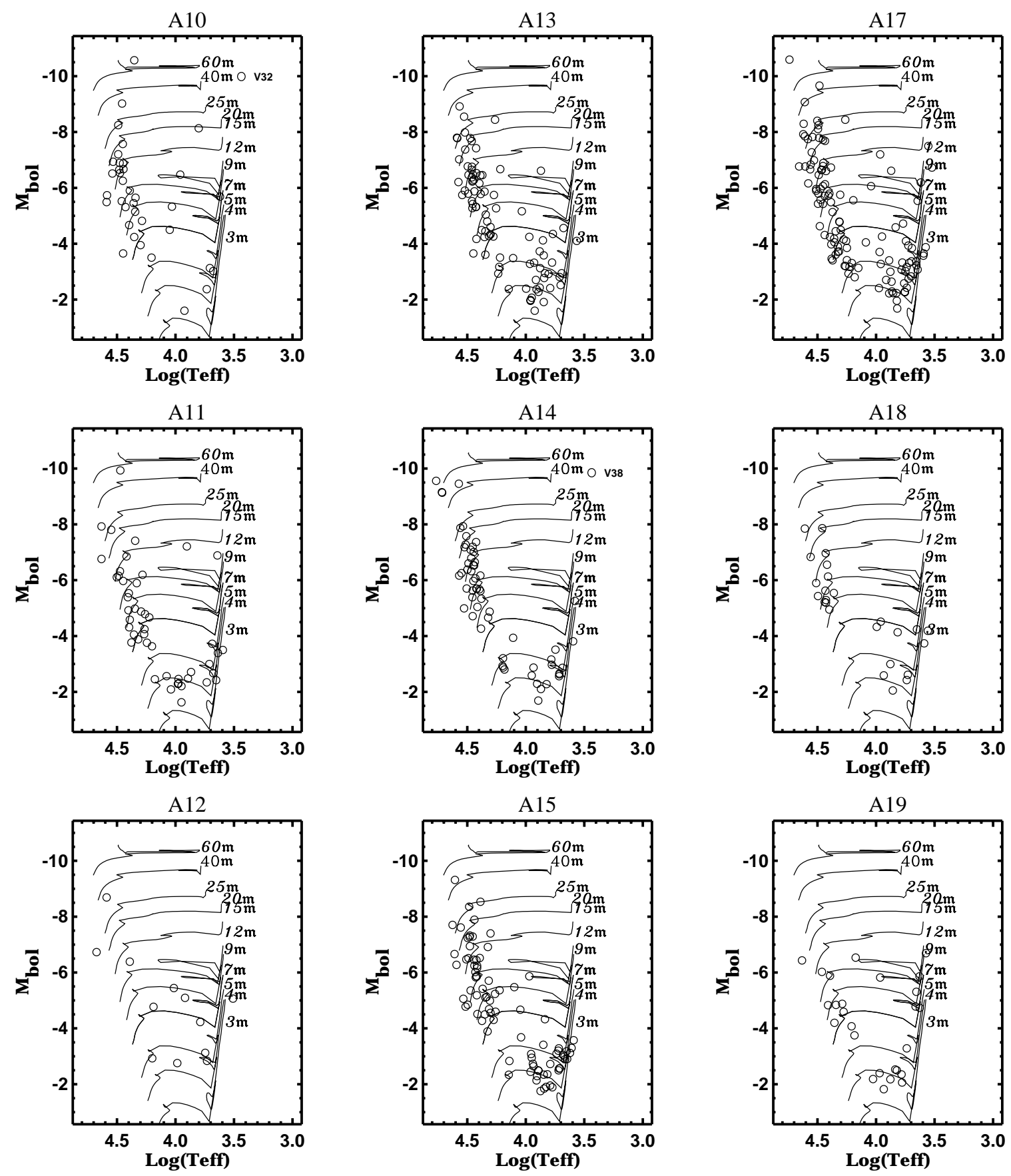

Fig. 13. Theoretical H-R diagrams for Hodge associations Nos. 10, 11, 12, 13, 14, 15, 17, 18 and No. 19

First group contains the blue stars from A10, A11, A14 and A17 (having ages between $5-8 \mathrm{Myr}$ ), second group contains A13 and A15 (having age 10 Myr) and the last one contains the stars from A12, A18 and A19 (with age $20 \mathrm{Myr}$ ). The slopes of $I M F s$ for these three groups are $\Gamma=-1.59 \pm 0.18, \Gamma=-2.22 \pm 0.20$ and $\Gamma=-2.65 \pm 0.05$ respectively. The uncertainty in the slope is the uncertainty in the least-squares fit to the $I M F$. According to Fabry Pérot images (Rosado 1998) the stars from the first group of Hodge associations are embedded into shells of gas, while there is not any gas in the last association group (A12, A18 and A19).

The different stellar content, age differences, variations of slopes of $I M F s$ and presence of gas into some of associations, show a signature of a presence of two waves of star formation. 

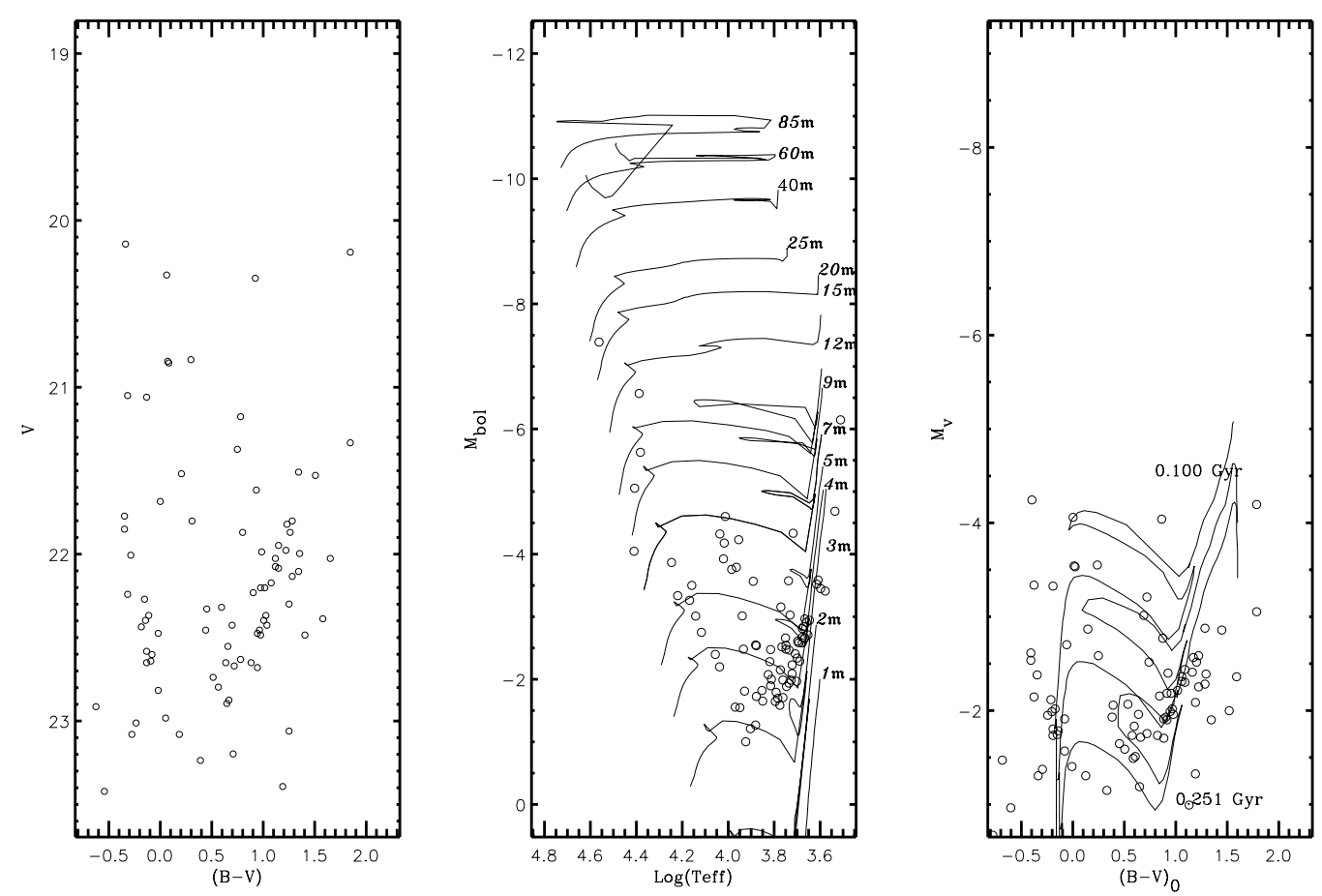

Fig. 14. The color-magnitude diagram, the isochrone comparison and the H-R diagram of the "comparison field area" with mean diameter $164 \mathrm{pc}$

Table 5. Parameters of the Hodge associations

\begin{tabular}{|c|c|c|c|}
\hline Name & Age(Myr) & $M_{\mathrm{up}}\left(M_{\odot}\right)$ & $N_{12} M_{\odot}$ \\
\hline A10 & 5 & 60 & 14 \\
\hline A11 & 8 & 50 & 9 \\
\hline A12 & 20 & 25 & 3 \\
\hline A13 & 10 & 40 & 28 \\
\hline A14 & 5 & 60 & 27 \\
\hline A15 & 10 & 40 & 28 \\
\hline A17 & 8 & 70 & 33 \\
\hline A18 & 20 & 20 & 7 \\
\hline A19 & 20 & 15 & 3 \\
\hline
\end{tabular}

\subsection{Criterion for the identification of $O B$ associations}

The stellar associations were identified by Hodge (1978) by eye estimation. The present criterion is the objective determination of the boundaries of $\mathrm{OB}$ associations. It suggests that OB stars should be assigned to one and the same stellar association if they have a statistically significant peak of surface stellar density above the mean level of the surrounding OB stars. The OB associations are groups with the highest stellar density. The neighbour distances between the stars inside the associations are substantially smaller than those of the background ones outside the association. The mean value of the distance to the nearest neighbour in an $\mathrm{OB}$ association is a measure for stellar density (see Appendix in Ivanov 1996). The stellar density function in the association is defined as $f\left(\delta_{j}\right)$, for $j=1,2, \ldots, N$, where $N$ is the number of candidate
OB associations in the galaxy. A reliable function for statistical evaluation of the possible maximal density of the $N$ stellar groups is the Fourier transform of the function $f\left(\delta_{j}\right)$ for the different candidates for OB associations:

$F\left(\nu_{k}\right)=\sum_{j}^{N} f\left(\delta_{j}\right) \mathrm{e}^{i 2 \pi s_{j} \nu_{k}}$,

where $s_{j}=(j-1)\left(d_{j}-d_{\lim }\right)$ is the interval of discretion of the mean neighbour distances for individual candidates for OB associations in the galaxy, $j, k=1,2, \ldots, N$, and $d_{\text {lim }}$ is a limiting search neighbour distance between stars which is a free parameter and corresponds to the highest possible stellar density inside the OB associations. The frequencies were defined as:

$\nu_{k}=\frac{k-1}{d_{\max }-d_{\lim }}$,

where $d_{\max }$ is the largest neighbour distance between the stars in the galaxy.

The amplitude of the Fourier transform is:

$$
\begin{aligned}
F F\left(\nu_{k}\right)=\frac{1}{N}\{ & {\left[\sum_{j}^{N} f\left(\delta_{j}\right) \cos \left(2 \pi s_{j} \nu_{k}\right)\right]^{2} } \\
& \left.+\left[\sum_{j}^{N} f\left(\delta_{j}\right) \sin \left(2 \pi s_{j} \nu_{k}\right)\right]^{2}\right\}^{1 / 2}
\end{aligned}
$$


Table 6. OB associations in IC 1613

\begin{tabular}{cccrrr}
\hline No. & $X$ & $Y$ & Size & Members & Density \\
\hline 1 & 979.8 & 642.6 & 33 & 5 & 22 \\
2 & 964.2 & 392.0 & 46 & 5 & 10 \\
3 & 937.7 & 716.3 & 35 & 5 & 24 \\
4 & 800.2 & 802.1 & 30 & 4 & 21 \\
5 & 775.9 & 417.1 & 27 & 5 & 28 \\
6 & 748.2 & 306.1 & 43 & 6 & 13 \\
7 & 707.0 & 486.8 & 129 & 36 & 13 \\
8 & 706.7 & 366.6 & 28 & 4 & 25 \\
9 & 675.0 & 667.0 & 33 & 4 & 17 \\
10 & 661.8 & 762.3 & 112 & 36 & 13 \\
11 & 640.7 & 565.8 & 92 & 14 & 6 \\
12 & 620.4 & 344.8 & 40 & 6 & 17 \\
13 & 595.2 & 143.9 & 42 & 6 & 14 \\
14 & 570.0 & 382.8 & 107 & 27 & 4 \\
15 & 569.2 & 249.4 & 112 & 50 & 21 \\
16 & 554.9 & 515.2 & 55 & 8 & 11 \\
17 & 537.6 & 557.1 & 31 & 7 & 40 \\
18 & 513.6 & 356.1 & 71 & 11 & 6 \\
19 & 486.7 & 683.3 & 51 & 6 & 8 \\
20 & 456.5 & 1003.6 & 53 & 7 & 11 \\
21 & 454.2 & 539.1 & 103 & 23 & 4 \\
22 & 444.4 & 869.7 & 86 & 10 & 4 \\
23 & 431.2 & 406.4 & 28 & 5 & 27 \\
24 & 388.7 & 443.4 & 45 & 5 & 10 \\
25 & 380.9 & 535.5 & 37 & 11 & 15 \\
26 & 368.8 & 28.4 & 40 & 5 & 12 \\
27 & 355.7 & 372.6 & 72 & 11 & 8 \\
28 & 324.1 & 618.0 & 111 & 15 & 12 \\
29 & 204.2 & 715.9 & 68 & 8 & 12 \\
30 & 18.20 & 320.4 & 41 & 8 & 19 \\
\hline & & & & &
\end{tabular}

Table 7. Data for some Hodge clusters and for two new cluster candidates

\begin{tabular}{lcccrcc}
\hline Cluster & $X$ & $Y$ & $V$ & $(B-V)$ & $(U-B)$ & \# ST \\
\hline C11 & 606.1 & 784.3 & 20.28 & -0.27 & -0.79 & 3 \\
C12 & 703.4 & 583.8 & 19.19 & 0.08 & -0.68 & 4 \\
C13 & 649.8 & 733.8 & 19.16 & -0.11 & -0.91 & 4 \\
C15 & 470.7 & 537.8 & 18.96 & -0.20 & -0.74 & 5 \\
C16 & 565.2 & 375.0 & 18.71 & -0.03 & -1.12 & \\
C17 & 538.1 & 289.4 & 18.12 & -0.13 & -0.84 & 5 \\
C20 & 758.0 & 169.4 & 20.60 & 0.34 & & \\
C40 & 585.4 & 407.5 & 18.22 & 0.01 & -1.02 & 6 \\
CL1 & 620.2 & 496.0 & 19.22 & -0.03 & -1.01 & 4 \\
CL2 & 533.2 & 407.5 & 19.38 & -0.08 & -0.97 & 4 \\
\hline
\end{tabular}

The OB associations have widely different stellar densities which is related to the mean neighbour distance for a given group $d_{j}$ as one can see from the relation (A6) of Ivanov (1996). In order to eliminate the random clumps of OB stars we introduce another free parameter, the minimum number of stars per OB association $N_{\min }$. The OB associations can be selected within some stellar densities varying the parameters $d_{j}$ and $N_{\text {min }}$. The function $F F_{\max }=\max F F\left(\nu_{k}\right)$ corresponds to the sum of stellar density fluctuations of the selected stellar groups
Table 8. Data for non-stellar objects

\begin{tabular}{lccccccc}
\hline No. & $X$ & $Y$ & $F W H M$ & $F W Q M$ & $V$ & $B-V$ & $U-B$ \\
\hline 1 & 190.98 & 212.98 & 4.96 & 7.00 & 19.04 & 1.65 & 0.74 \\
2 & 619.21 & 389.52 & 5.32 & 7.46 & 19.89 & 1.37 & \\
3 & 863.83 & 549.11 & 5.78 & 8.30 & 19.51 & 1.15 & 0.71 \\
4 & 653.02 & 638.79 & 5.48 & 7.64 & 19.59 & 1.49 & 0.54 \\
5 & 518.32 & 745.98 & 5.10 & 7.24 & 19.62 & 1.25 & 0.44 \\
6 & 879.18 & 927.53 & 6.24 & 8.80 & 19.77 & 0.92 & $-0.09:$ \\
\hline
\end{tabular}

above the local OB stellar background which is the highest. The maximum of function $F F_{\max }$ was obtained for $d_{j}=20.4 \mathrm{px} \approx 7 \operatorname{arcsec}$ and $N_{\min }=4$.

These parameters select the smallest groups in IC 1613 with a mean size $(\approx) 60$ pc. These 30 groups have the smallest size in the hierarchy of $\mathrm{OB}$ stellar groups but they have the highest stellar density in IC 1613.

Following the present criterion we select relatively small groups with high stellar density which is at least 4 times higher than that of the surrounding OB stars. However the mean stellar density of the 30 selected groups is 35 times above the background OB stars.

The re-determined OB associations are shown in Table 6. Column 1 gives the number of the group, Cols. 2 and 3 give the mean coordinates of the group in pixels, Col. 4 gives the average size of the group in pc, Col. 5 gives the number of member stars and column 6 gives the mean stellar density. A map of the new associations is shown in Fig. 15.

Most of the stellar associations outlined by Hodge coincide with our groups but the present criterion divides Hodge's associations into several smaller groups. They look like bright cores within Hodge's associations. The present criterion selects a core structure of OB stars in the classic associations. They are probably the youngest OB stars in the associations. It is known that young associations in the Galaxy like Trapezium contain small compact subgroups similar to those selected by the present criterion in IC 1613 while the older associations consist of randomly distributed stars and they could not be divided into subgroups. Using the present criterion we identified 30 small groups of OB stars with a mean size of about $60 \mathrm{pc}$. Hodge (1986) describes the problem of the different sizes of the stellar associations in the Magellanic clouds and other nearby galaxies as M31 and M33. He concluded that the mean size of the associations depends on the plate scale and the distance to the galaxy. The present criterion is very strong, the result being that only the youngest associations are identified. This may explain the smaller mean size of the stellar groups identified in the present paper than those presented by Hodge (1986). 


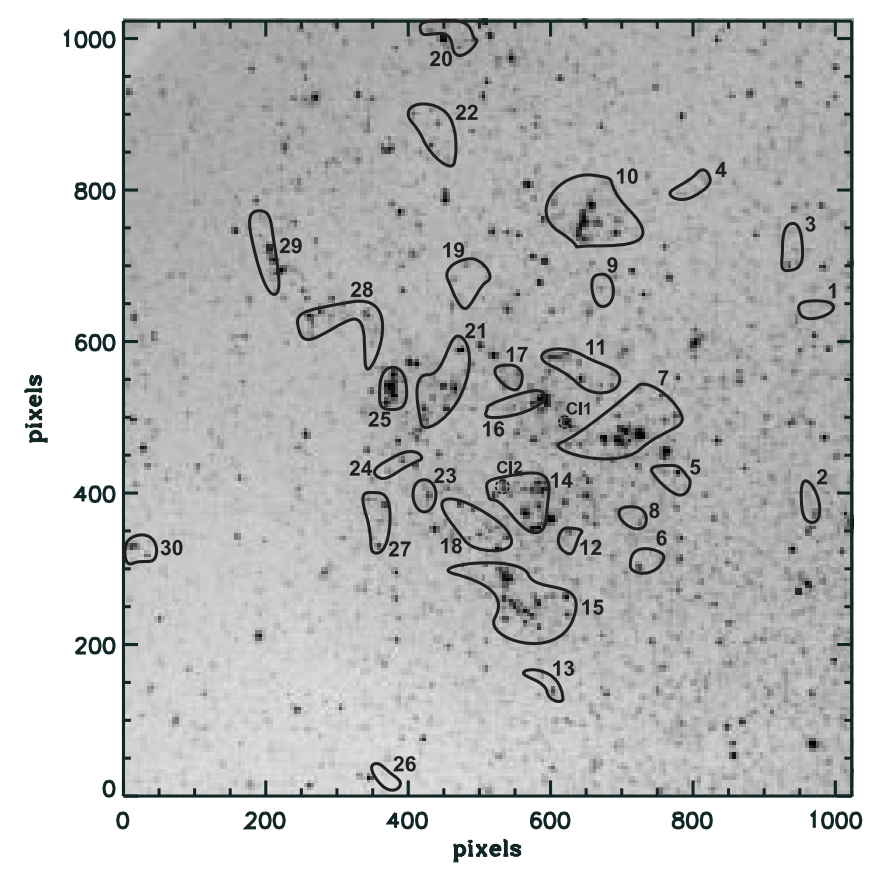

Fig. 15. Observational area for IC 1613 with superimposed boundaries of new associations. The two new open cluster candidates are marked by dashed line.North is on top, East is to the left

\section{Clusters and non-stellar objects}

\subsection{Hodge open clusters}

Little progress has been made in this area since the original suggestion of Baade (1963) that the number of clusters in IC 1613 is very low. Hodge (1978) identified by visual inspection 43 cluster candidates. Except for a number of cluster stars (usually $4-5$ up to $V=23 \mathrm{mag}$ ) there is no other information about them.

We identified twelve Hodge clusters in our field of observation. Growth curves aperture photometry was applied to eight of them in order to determine the total $U$ (except for C20), $B$ and $V$ magnitudes (Table 7). Local sky background reaches up to diaphragms of about $7 \operatorname{arcsec}(\approx 24 \mathrm{pc})$. This is not much above the diameters given by Hodge $(\approx 17 \mathrm{pc})$. The only exception is C40, noted by Hodge as "loose", with a diameter of 10 arcsec ( $\approx 34 \mathrm{pc})$. We have $U B V$ photometry for the brightest stars of each cluster. The integrated magnitudes and colors of the brightest cluster members differ slightly from the total aperture magnitudes and colors.

The $(U-B)$ vs. $(B-V)$ diagram is presented in Fig. 16. The observed distribution of clusters in the colorcolor $(U-B, B-V)$ diagram can be related to the SWB classification which was originally based on the Gunn photometric system (Bica et al. 1996). It is basically an age sequence. Six clusters from our sample fall into the SWB 0 type zone (Bica et al. 1992) for very young clusters associated with the HII regions and ages between 0 and
10 Myr. Only C 12, noted by Hodge as "red giants", falls into the SWB 1 type zone of clusters around $10 \mathrm{Myr}$. The integrated colors of the clusters belonging to this group may reach very red $B-V$ colors owing to a red supergiant phase.

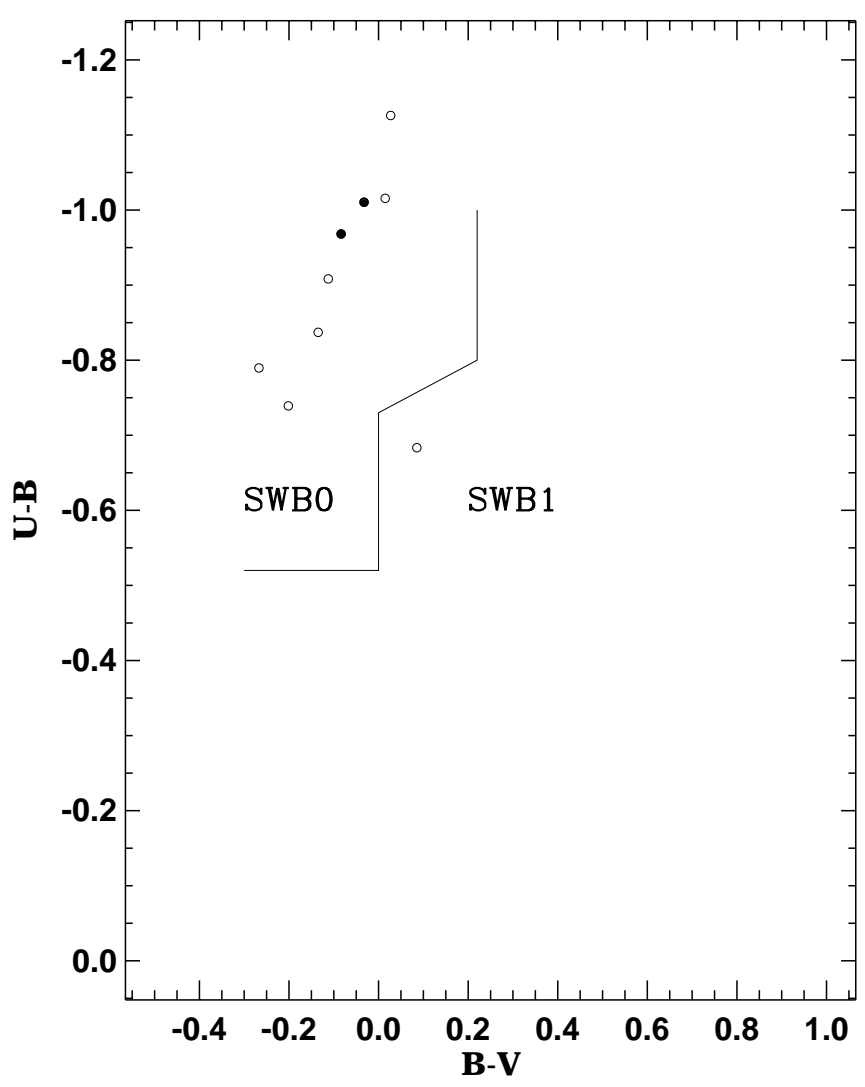

Fig. 16. $(U-B)$ vs. $(B-V)$ diagram for Hodge clusters. The border line between SWB 0 and SWB 1 zones is shown. Two new cluster candidates are marked by filled circles

Two new cluster candidates are identified using the method described above. The magnitudes and colors for them are given in Table 7 . The new cluster candidates also fall into the SWB 0 type zone (Bica et al. 1992) for very young clusters (marked in Fig. 16 by filled circles).

\subsection{Non-stellar objects}

There is a remarkable lack of globular clusters in IC 1613 (Hodge 1978; Freedman 1988a). This fact was a prompt to carry out a new search for globulars. All objects with $\chi^{2}>1.5$ were derived from our ALLSTAR photometry lists in $B V$. Six most probable nonstellar objects were selected after a careful "object by object" inspection of the resultant profiles and a thorough check for unresolved close neighbours. Following Crampton et al. (1985) the full widths of the half-maximum (FWHM) and the quarter maximum (FWQM) were measured for these objects 
as well as for a representative sample of 30 randomly scattered and well resolved stars. A plot of FWHM vs. FWQM (in pixels) for the $V$ frame is presented in Fig. 17. It shows a clear separation between the sample stars (filled circles) and the non-stellar objects (open circles). We then made an additional growth curves aperture photometry for non-stellar candidates (Table 8). All objects are relatively bright but red. Taking into account the very low reddening towards IC 1613 we conclude that they can not be globulars which confirms the lack of globular clusters in this galaxy. According to Fabry Pérot images (Rosado 1998) these are no peculiar velocities or emissions around these nonstellar objects. We can only speculate that these objects are perhaps stars embedded into shells of dust but most probably they are distant galaxies visible through the disk of IC 1613.

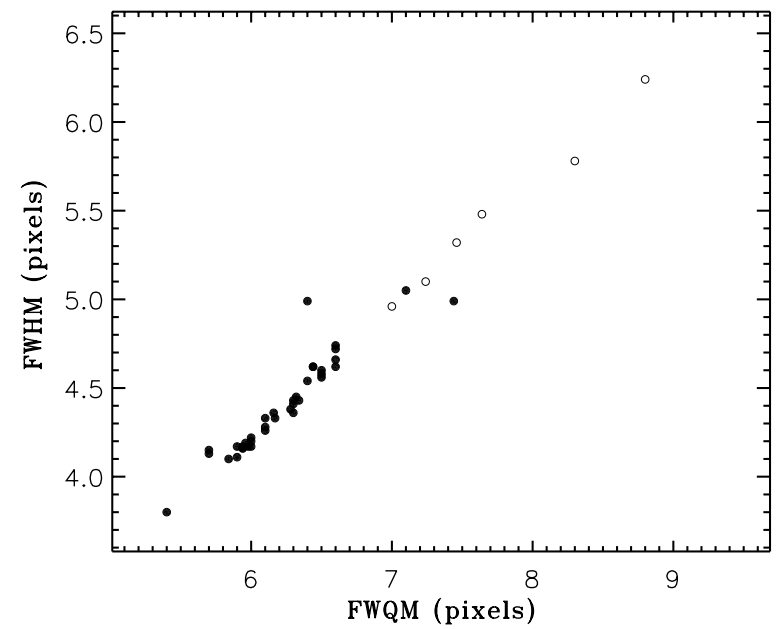

Fig. 17. The FWHM vs. FWQM plot for nonstellar objects (open circles) and sample stars (filled diamonds)

\section{Summary}

1. We have presented first deep $U B V$ photometry of the Dwarf Irregular galaxy IC 1613 in the Local Group. The selected area is a $5.6 \times 5.6$ arcmin field centered on the HII regions in the northeast sector of the galaxy.

2 . The blue color-color $(U-B, B-V)$ diagram was constructed for the first time making it possible to determine the differential reddening $E(B-V)=0.06$ with $\sigma=0.02$

3. The isochrones fit clearly shows the presence of stars from a wide variety of ages. The ages of the blue stars are between 5 and 20 Myrs. Most of the yellow to red stars fainter than $M_{V}=-4.0$ are roundly matched by the post main-sequence part of the 60 Myrs to 250 Myrs isochrones. The old age population was reliably detected thus confirming Freedman's (1988a) suggestion for the si- multaneous presence of young, intermediate-age and old populations.

4. The theoretical $\mathrm{H}-\mathrm{R}$ diagram has been constructed. The most massive stars in this area of IC 1613 reach $85 M_{\odot}$. The young stars closely follow the ZAMS. The red part of the $\mathrm{H}-\mathrm{R}$ diagram contains some evolved red supegaints between 15 and $40 M_{\odot}$ and a large amount of low-mass yellow and red giants. We determined $\log \left(T_{\text {eff }}\right)$ and $M_{\mathrm{bol}}$ for all variable stars available in the field and pointed out the peculiar position of two very blue irregulars - V8 and V21.

5 . We determined the $I M F$ for the blue stars of equal age. The $I M F$ slope is $\Gamma=-2.0$ with $\sigma=0.1$. The comparison with the IMF slopes of other LMC, SMC and M 31 associations displays no differences within the error range and probably the slope of the $I M F$ is really constant (Massey et al. 1995a,b).

6. Nine of the associations outlined by Hodge (1978) were investigated. The ages of the associations were determined by means of an isochrone fit using the method of Flannery \& Johnson (1982) on the assumption that within each association all stars are born coeval. A10 and A14 were found to be the youngest associations with ages of approximately $5 \mathrm{Myr}$, while A12, A18 and A19 were the oldest ones found, having a common age of approx. 20 Myr. Hodge included the brightest red variable supergiants V32 and V38 in the boundaries of A10 and A14 respectively (Sandage 1971). Taking into account their masses, $M_{\text {bol }}$ and $\log \left(T_{\text {eff }}\right)$ as well as the derived age of A10 and A14 we conclude from the results in Massey's paper (1998) that they could be real members of those associations. The comparison with the red supergaints in M 31, M 33 and NGC 6822 discussed in the same paper shows that the two supergaints in IC 1613 are similar to the red supergaints in NGC 6822.

7. The OB stars in our field were re-grouped by cluster analysis. Our present criterion generally divides a Hodge association into several smaller groups thus defining a total of 30 high-density groups. 23 of them cover the Hodge outlines and 7 are completely new ones.

8. Eight Hodge open clusters were identified in our field of observations. Two new cluster candidates were obtained by cluster analysis. The total $U, B$ and $V$ magnitudes were determined for the first time. The observed distribution of clusters in the color-color $(U-B, B-V)$ diagram was compared with the SWB classification of Bica et al. (1996). Eight clusters from our sample fall into the SWB 0 type zone (Bica et al. 1992) for very young clusters associated with the $\mathrm{HII}$ regions and ages between 0 and 10 Myr. Only C 12 falls into the SWB 1 type zone of clusters around $10 \mathrm{Myr}$. The integrated colors of the clusters belonging to this group may reach very red $B-V$ colors owing to a red supergiant phase.

9. Six new possible nonstellar objects are reported. Most probably they are distant galaxies visible through 
the disk of IC 1613 which confirms Baade's original suggestion that IC 1613 contains no bright globular clusters.

Acknowledgements. L.G., J.B. and R.K. would like to thank T. Valtchev and A. Staneva for their help in the process of obtaining the CCD frames and also E. Chelebiev for his help. The authors gratefully acknowledge the useful comments and suggestions raised by Dr. V.D. Ivanov and Dr. N. Spassova, as well as the comments by an anonymous referee. This research was supported by the "Programa de cooperations MexicoRepublica de Bulgaria - "Estrellas Masivas en las Galaxias del Grupo Local" Of. No. 403. S.T.A.C.R.I.25/97. This work was performed while J.B. was a visiting astronomer in UNAM, Mexico under contacts CONACYT No. 400354-5-2398PE and DGAPA INI04696.

\section{References}

Armandroff T., Massey P., 1985, ApJ 291, 685

Baade W., 1928, Astr. Nach 234, 407

Baade W., 1963, In Evolution of Stars and Galaxies. Harvard University, Cambridge, MA

Bahcall J.N., Soneira R.M., 1980, ApJS 44, 173

Bencivenni D., Brocato E., Buonanno R., Castellani V., 1991, AJ 102, 137

Bertelli G., Bressan A., Fagotto F., Choisi C., Nasi E., 1994, AAS 106, 275

Bica E., Clariá J., Dottori H., 1992, AJ 103, 1859

Bica E., Clariá J., Piatti A., 1996, ApJS 102, 57

Gallart C., Aparicio A., Vilchez J.M., 1996a, AJ 112, 1928

Gallart C., Aparicio A., Bertelli G., Chiosi C., 1996b, AJ 112, 1950

Gallart C., Aparicio A., Bertelli G., Chiosi C., 1996c, AJ 112, 2596

Carlson G., Sandage A., 1990, ApJ 352, 587

Charbonnel C., Maynet G., Maeder A., Schaller G., Schaerer D., 1993, AAS 101, 415

Crampton D., Cowley A., Schade D., Chayer P., 1985, ApJ 288,494

Flannery B., Johnson B., 1982, ApJ 263, 166

Flower P., 1996, ApJ 469, 355

Freedman W., 1988a, AJ 96, 1248

Freedman W., 1988b, ApJ 326, 1988

Ivanov G., 1996, AA 305, 708
Johnson H., Morgan W., 1953, ApJ 117, 313

Haiman Z., Magnier E., Battinelli P., Lewin W., et al., 1994, AA 290, 371

Helfand D., 1996, Technical Report, Columbia Univ. New York, NY United States

Hodge P., 1978, ApJS 37, 145

Hodge P., 1986, in de Loore, A. Willis and Laskarides (eds.), "System of Stellar Associations in Galaxies", IAU Symp. 132,369

Hodge P., Smith T., Eskridge P., Macgillivray H., Beard S., 1991, ApJ 369, 372

Kingsbugh R., Barlow M., 1995, AA 295, 171

Lake G., Skillman E., 1989, AJ 98, 127

Massey P., Garmany C., Degiola-Eastwood K., 1989, AJ 97, 107

Massey P., Lang C., DeGioia-Eastwood K., Garmany C., 1995a, ApJ 438, 188

Massey P., Johnson K., DeGioia-Eastwood K., 1995b, ApJ 454, 151

Massey P., 1998, NOAO preprint No. 778

Parker J., Garmany C., Catharine D., Massey P., Walborn N., 1989, AJ 103, 1205

Parker J., Garmany C., 1993, AJ 106, 1471

Peimbert M., Bohigas J., Torres-Peimbert S., 1988, Rev. Mexicana Astron. Astrof. 16, 45

Rosado M., 1998 (private communication)

Phelps R., Janes K., 1993, AJ 106, 1870

Ratnatunda K., Bahcall J., 1985, ApJS 58, 63

Reimann H., Pfau W., 1987, Astron. Nachr. 308, 111

Sandage A., 1971, ApJ 166, 13

Saha A., Freedman W., Hoessel J., Mossman A., 1992, AJ 104, 1072

Schmidt-Kaler T., 1982, in Landolt-Bornstein, Group VI, Vol. 2: Astronomy and astrophysics, Subvolume b: Star and star clusters, Schaifers K. and Voigt H.H. (eds.). Springer, Berlin

Stetson P., Harris W., 1988, AJ 96, 909

Stetson P., 1991, in The Formation and Evolution of Star Clusters, ASP Conf. Ser. 13, Janes K.A. (ed.). ASP, San Francisco, p. 88

Stetson P., 1993, Users manual for DAOPHOT II

Westpfahl D., Wilcots E., Graham M., Olsen K., 1996, AAS 188,4316

Wolf M., 1906, MNRAS 67, 91

Vanden Berg D., 1997 (private communication) 